\title{
Cardiomyocyte-Derived Exosomes: Biological Functions and Potential Therapeutic Implications
}

\author{
Hui Yu and Zhanli Wang* \\ The Second Affiliated Hospital, Baotou Medical College, Baotou, China
}

Exosomes, which are membrane-enclosed nanovesicles released by almost all cell types, have been recognized to play important roles in mediating cell-cell communication. In recent years, the physiological and pathological effects of exosomes on cardiovascular disease have been extensively studied. Exosomes can transfer proteins, mRNAs, microRNAs, and other bioactive molecules to recipient cells to influence their biological properties. In recent years, accumulating evidence has suggested that cardiomyocyte-derived exosomes play an important role in the progression of cardiovascular disease. Here, we summarize the functional roles of cardiomyocyte-derived exosomes in cardiovascular physiology and pathology.

Keywords: cardiomyocyte, exosome, biological function, cardiovascular disease, therapeutic application

\section{INTRODUCTION}

Heart disease, or CVD, has become the most common cause of mortality globally (Lai et al., 2019). Major types of heart disease include coronary artery disease, stroke, heart failure, hypertensive heart disease, rheumatic heart disease, cardiomyopathy, inflammatory heart disease, and others (GBD 2013 Mortality and Causes of Death Collaborators, 2015). The heart consists of a complex mixture of various cell types, including cardiomyocytes, fibroblasts, endocardial and epicardial cells, inflammatory cells, and immune cells. These cells within the heart communicate intensively to facilitate proper cardiac function through direct cell-cell contact and paracrine interactions (Talman and Kivelä, 2018).

Exosomes, which were first identified by Johnstone et al. (1987), are small membrane-bound vesicles with a diameter of 30-100 $\mathrm{nm}$. Exosomes are secreted by most cell types, including lymphocytes, platelets, and adipocytes, as well as tumor, muscle, and stem cells (Brisson et al., 2017; Barlow and Solomon, 2018; Meng et al., 2019; Scherer, 2019; Seo et al., 2019; Torralba et al., 2019). Exosomes mediate intercellular signaling and communication by shuttling nucleic acids, proteins, and lipids between cells; knowledge of their functions can provide novel diagnostic and

Abbreviations: Ang II, Angiotensin II; ATG12, autophagy-related gene 12; BMSCs, bone marrow mesenchymal stem cells; CCL, chemokine ligand; CVD, cardiovascular disease; ERK1/2, extracellular signal-regulated kinase 1/2; ESCRT, endosomal sorting complexes required for transport; GLUT, glucose transporters; Hsp, heat shock protein; IGF-1, insulin-like growth factor-1; ILV, intraluminal vesicle; IL-6, interleukin-6; LC3, microtubule associated protein light chain 3; miRNA, microRNA; MMP9, matrix metalloprotein 9; mRNA, messenger RNA; mTOR, mammalian target of rapamycin; MVB, multivesicle body: Nrf2, nuclear factor erythroid-2 related factor 2; PDGF, platelet-derived growth factor; p38 MAPK, p38 mitogen-activated protein kinase; TGF- $\beta$, transforming growth factor beta; TNF- $\alpha$, tumor necrosis factor- $\alpha$. 
therapeutic strategies for many diseases (Fruhbeis et al., 2012; You et al., 2018; Wu et al., 2019). Similarly, in the heart, exosomes can act as vehicles to deliver cargo to neighboring or distant cells to reprogram the cardiac microenvironment (Kishore et al., 2016; Juni et al., 2017; Marini et al., 2017; Poe and Knowlton, 2017). Indeed, exosomes play a substantial role in various processes involved in the pathogenesis of CVDs such as cardiac fibrosis, hypertrophy, myocardial apoptosis, and angiogenesis (Bei et al., 2017a; Salem and Fan, 2017; Davidson and Yellon, 2018). In this review, we summarize current knowledge regarding cardiomyocyte-derived exosomes and their applications in the diagnosis and possible repair of cardiac damage.

\section{BIOGENESIS OF CARDIOMYOCYTE-DERIVED EXOSOMES}

The generation of exosomes is associated with the endosomal network (Stoorvogel et al., 2002; Akers et al., 2013; Frydrychowicz et al., 2015; Figure 1). Initially, the inward budding of the cell membrane forms early endosomes, which are embedded with specific membrane proteins. Subsequently, various ILVs are generated by further inward budding of the endosomal membrane; these early endosomes are known as multi-vesicle bodies (MVBs). Finally, the MVBs fuse with lysosomes and release ILVs for degradation or recycling, or they fuse with the plasma membrane and release the ILVs as exosomes. Endosomal Sorting Complex Required for Transport (ESCRT)dependent and -independent mechanisms participate in the biogenesis of exosomes in MVBs (Jakhar and Crasta, 2019). The ESCRT-dependent pathway, which includes the distinct subcomplexes ESCRT-0, ESCRT-I, ESCRT-II, and ESCRTIII, directs the sorting of ubiquitinated proteins and is responsible for the inward budding of ILVs into the interior of endosomes, thereby forming MVBs (Kowal et al., 2014). The ESCRT-independent pathway in the biogenesis of exosomes has been found to require the participation of the Golgi apparatus to package proteins into exosomes within MVBs (Trajkovic et al., 2008).

The generation and release of exosomes derived from cardiomyocytes is influenced by many factors. Previous findings showed that Hsp20 mediated the activation of exosome biogenesis and their secretion from cardiomyocytes by interacting with Tsg101, a major exosome biogenesis mediator (Wang et al., 2016). Cardiomyoblasts also display increased exosome excretion under glucose starvation (Garcia et al., 2015). Loyer et al. (2018) demonstrated that myocardial infarction triggers the release of cardiomyocyte-derived exosomes. Additionally, previous reports have demonstrated that ethanol treatment increased exosome formation and secretion from cardiomyocytes (Malik et al., 2013). Furthermore, treatment with TGF- $\beta$ and PDGF also affected the characteristics of biogenesis and the release of exosomes in cultured cardiomyocytes (Gennebäck et al., 2013). Moreover, under stresses such as increased Ang II production, hypoxia, inflammation, or injury, cardiomyocytes have been found to display increased exosome secretion (Chistiakov et al., 2016; Yang et al., 2018).

\section{THE REPORTED MOLECULAR CONTENTS OF CARDIOMYOCYTE-DERIVED EXOSOMES}

To date, numerous studies have indicated that exosomes carry an extensive range of biomolecules including proteins, lipids, carbohydrates, mitochondrial DNA (mtDNA), mRNAs, miRNAs, and long non-coding RNAs (lncRNAs). However, the composition of exosomes is influenced by the cell of origin and the pathophysiological status leading to exosome formation, suggesting the potential functional diversity of exosomes (Katsuda et al., 2014; Conigliaro et al., 2017; Cheng et al., 2018). Recently, a large variety of biomolecules have been identified in exosomes secreted from cardiomyocytes in several studies. Expectedly, the presence of proteins within exosomes has been consistently shown in recent reports (Table 1). Hsps play essential roles in cellular survival and adaptation under numerous stresses (Gupta and Knowlton, 2007). An increasing number of observations have shown that cardiomyocyte-derived exosomes are enriched for Hsps (Hsp20, Hsp60, and Hsp70) involved in regulating cardiomyocyte growth and survival under stress (Gupta and Knowlton, 2007; Zhang et al., 2012; Malik et al., 2013; Feng et al., 2014; Yu et al., 2019). Recent findings have also demonstrated that myocyte-derived exosomes contain inflammatory factors such as IL-6 and TNF- $\alpha$, which are responsible for cardiac remodeling (Yu et al., 2012; Datta et al., 2017). Furthermore, exosomes derived from cardiomyocytes were found to carry functional GLUT (GLUT4, GLUT1) and glycolytic enzymes (lactate dehydrogenase), and were shown to have specialized functions in glucose transport and metabolism in endothelial cells (Garcia et al., 2016). Malik et al. (2013) investigated the protein contents of different exosome extracts after ethanol or hypoxia/reoxygenation treatment by mass spectrometry. They identified 51 different proteins in exosomes derived from ethanol-treated cells, and 33 proteins in those derived from hypoxia/reoxygenationtreated cells, ranging from membrane-bound to cytosolic and mitochondrial proteins.

Many studies have demonstrated that exosomes derived from cardiomyocytes can also carry nucleic acid cargo. Waldenström et al. (2012) identified 343 different sequences of chromosomal DNA in microvesicles/exosomes derived from the cultured cardiomyocytes. Wang et al. (2014) reported that exosomes secreted from the cardiomyocytes of diabetic patients carried higher levels of miR-320 and lower levels of miR-126 compared to those secreted from healthy cardiomyocyte-derived exosomes. Exosomal miR-320 exerts an anti-angiogenic function by suppressing its target genes in recipient endothelial cells, including Hsp20, V-ets erythroblastosis virus E26 oncogene homolog 2 (Ets2), and IGF-1. Recent studies also indicated that 


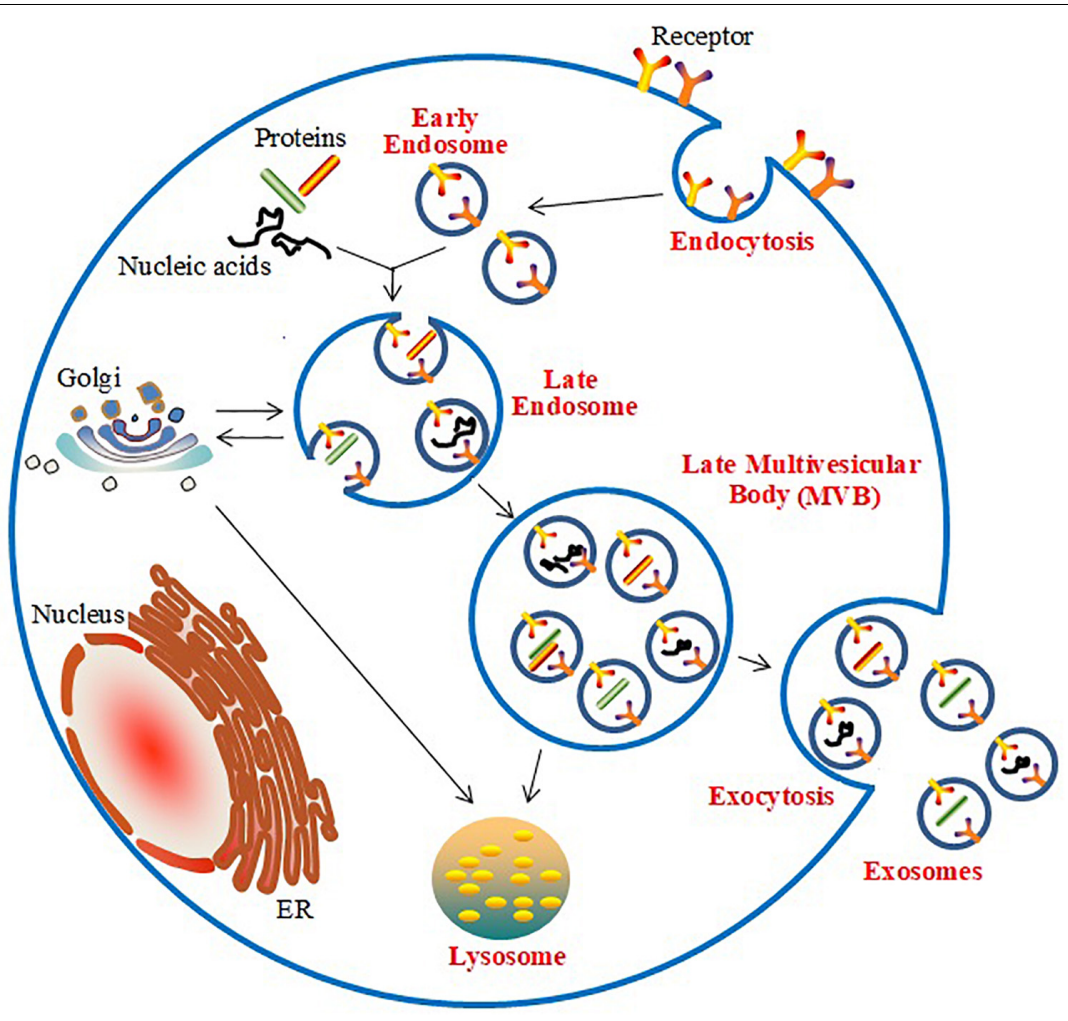

FIGURE 1 | Biogenesis and release of exosomes.

miR-30a was present at high levels in hypoxic cardiomyocytederived exosomes (Yang et al., 2016). Chaturvedi et al. (2015) further found that exosomes from cardiomyocytes were enriched for certain miRNAs (particularly miR-29b, miR-323-5p, miR455, and miR-466) and mediated the regulation of MMP9 expression in diabetic heart tissues. Additionally, miR-27a, miR28-3p, miR-34a, and miR-208a were found to be highly expressed in cardiomyocytes and preferentially incorporated into exosomes (Tian et al., 2018; Yang et al., 2018). Table 2 shows the major reported cardiomyocyte-derived exosomal miRNAs and their biological functions. As carriers of various biomolecules, exosomes protect their cargo against digestion by enzymes present in the body fluid. Therefore, the encapsulated contents can be transported to their extracellular destinations (Katsuda et al., 2014; Ohno and Kuroda, 2016).

\section{ISOLATION OF CARDIOMYOCYTE-DERIVED EXOSOMES}

Exosome isolation is a fast-growing field of study. The efficient isolation of high-quality exosomes is critical for determining their applications in biomedical sciences. However, since exosomes are very small vesicles, there are several technical issues impeding their successful isolation (Gurunathan et al., 2019). One challenge is the absence of suitable techniques for accurate exosome
TABLE 1 | The reported proteins within cardiomyocyte-derived exosomes.

\begin{tabular}{|c|c|c|c|}
\hline $\begin{array}{l}\text { Exosomal } \\
\text { cargo }\end{array}$ & Expression & Biological function & References \\
\hline Hsp20 & Increased & $\begin{array}{l}\text { Promote angiogenesis; } \\
\text { Improve cardiac function }\end{array}$ & $\begin{array}{l}\text { Zhang et al., 2012; Yu } \\
\text { et al., } 2019\end{array}$ \\
\hline Hsp60 & Increased & $\begin{array}{l}\text { Promote immune } \\
\text { responses }\end{array}$ & $\begin{array}{l}\text { Gupta and Knowiton, } \\
2007\end{array}$ \\
\hline Hsp70 & Increased & Improve cardiac function & Feng et al., 2014 \\
\hline TNF- $\alpha$ & Increased & $\begin{array}{l}\text { Contribute to cardiac } \\
\text { remodeling }\end{array}$ & Yu et al., 2012 \\
\hline IL-6 & Increased & $\begin{array}{l}\text { Contribute to cardiac } \\
\text { fibroblasts }\end{array}$ & Datta et al., 2017 \\
\hline GLUT1 & Increased & Regulate metabolism & Garcia et al., 2016 \\
\hline GLUT4 & Increased & Regulate metabolism & Garcia et al., 2016 \\
\hline
\end{tabular}

characterization. Currently, various techniques for the isolation of exosomes have been reported, such as ultracentrifugation (Van Deun et al., 2014), size-based filtration (Zeringer et al., 2015), size-exclusion chromatography (Momen-Heravi et al., 2012), polymer precipitation (Ibrahim et al., 2014), immunoaffinity purification (Zarovni et al., 2015), and microfluidicsbased isolation techniques (Wang et al., 2013). Each approach has its disadvantages and advantages. Ultracentrifugation at high speeds can be used to obtain a highly pure exosomal fraction from cell culture media. However, this method is not suitable for small volumes of clinical samples with complex mixtures containing 
TABLE 2 | The reported miRNAs within cardiomyocyte-derived exosomes.

\begin{tabular}{llll}
\hline $\begin{array}{l}\text { Exosomal } \\
\text { cargo }\end{array}$ & Expression & Biological function & References \\
\hline miR-320 & Increased & Inhibit angiogenesis & Wang et al., 2014 \\
miR-30a & Increased & Regulate autophagy & Yang et al., 2016 \\
miR-29b & Increased & $\begin{array}{l}\text { Inhibit fibrosis and } \\
\text { myocyte uncoupling }\end{array}$ & Chaturvedi et al., \\
& & Inhibit fibrosis and & Chaturvedi et al., \\
miR-455 & Increased & myocyte uncoupling & 2015 \\
miR-27a & Increased & $\begin{array}{l}\text { Contribute to oxidative } \\
\text { stress }\end{array}$ & Tian et al., 2018 \\
miR-28-3p & Increased & $\begin{array}{l}\text { Contribute to oxidative } \\
\text { stress }\end{array}$ & Tian et al., 2018 \\
miR-34a & Increased & Contribute to oxidative & Tian et al., 2018 \\
& & stress & \\
miR-208a & Increased & Promote fibroblast & Yang et al., 2018 \\
& & proliferation & \\
\hline
\end{tabular}

a large number of components, because it is difficult to remove other contaminating membrane vesicle populations using this method (Witwer et al., 2013). Size-based filtration methods are not suitable for the enrichment of exosomes (Gurunathan et al., 2019). It must be emphasized that size exclusion chromatography and immuno-affinity purification cannot be used to discriminate larger fragmented microparticles from exosomes, leading to the impurity of yields (György et al., 2011; Peterson et al., 2015). Recent studies have suggested that immune affinity capture is a specific technique to isolate exosomes; however, yields are generally low from this method (Prunotto et al., 2013). In addition to these traditional isolation techniques, many commercial kits for the isolation of exosomes are available, such as ExoQuick Exosome Precipitation Solution, Total Exosome Isolation, and exoRNeasy Serum/Plasma Kit (Macías et al., 2019). ExoQuick Exosome Precipitation Solution and Total Exosome Isolation are convenient precipitation solutions, which have been utilized to precipitate particles in liquid, but they are unable to resolve particle heterogeneity (Lobb et al., 2015). The exoRNeasy Serum/Plasma kit was used for isolation of exosomes using a membrane-based affinity binding step from serum or plasma (Enderle et al., 2015). Recently, Malik et al. (2016) reported a simple, low-cost, and effective method for the isolation of exosomes derived from cardiac myocytes. In brief, cardiac myocytes are cultured in Media 199 for $2 \mathrm{~h}$, and the medium is then replaced with albumin-free Media 199. Next, cardiac myocytes are treated with cell culture-grade ethanol or with hypoxia-inducing factors. After treatment, the media is collected and centrifuged at $300 \times g$ for $10 \mathrm{~min}$, followed by centrifugation at $4,700 \times g$ for $30 \mathrm{~min}$. The resulting supernatant is then collected and concentrated. Next, ExoQuick is used to complete the isolation of the exosomes. Their study further evaluated the reliability and effectiveness of this approach in terms of the purity, size, morphology, and proteome content of extracted exosomes. Their paper further confirmed the high quality and reliability of this method, which was shown to be efficient, reliable, and reproducible. However, this approach for the purification of cardiac myocyte exosomes is limited to conditioned media. The water, tubing and ethanol used for the cardiac myocytes isolation must be cell culture grade, avoiding the cardiac myocytes damage. It is also very important to perfuse the isolated heart in order to obtain as many living cells as possible. Moreover, the adult cardiac myocytes should be treated with ethanol to increase exosome production and the suitable ethanol concentration was very important.

Moreover, researchers rely on the identification of certain exosomal markers to confirm the presence of exosomes (Mathivanan and Simpson, 2009). The tetraspanin CD63 and tumor susceptibility gene protein 101 (TSG101) are common exosomal marker proteins found in cell suspension (Gennebäck et al., 2013). The tetraspanins CD9 and CD81 are also molecular markers of cardiomyocyte-derived exosomes (Wang et al., 2014; Garcia et al., 2015; Yang et al., 2018). Moreover, Hsp70 is a specific marker of cardiomyocyte-derived exosomes, and can be identified by western blotting (Kuo et al., 2019). However, exosomes formed through ESCRT-independent pathways are usually devoid of biomarkers associated with the ESCRT complex, such as CD63 or CD9 (Akers et al., 2013). To rule out the contamination of other membrane vesicle populations, researchers have often performed the simultaneous detection of endoplasmic reticulum-related markers such as calnexin and glucose-regulated protein 78 (Grp78) (Gennebäck et al., 2013; Kuo et al., 2019).

\section{CARDIOMYOCYTE-DERIVED EXOSOMES AND CARDIOVASCULAR CELL-CELL COMMUNICATION}

In addition to other extracellular molecules that can mediate intercellular communication, increasing attention has been given to exosomes (Pegtel et al., 2010; Cervio et al., 2015). Exosomes have become an important player in intercellular signaling via several types of interaction pathways, including delivering their molecular cargo into the target cell, directly regulating membrane receptors of the target cell, and changing the microenvironmental milieu of the target cell (Sluijter et al., 2014; Poe and Knowlton, 2018; Figure 2). Waldenström et al. (2012) demonstrated that cardiomyocyte-derived exosomes packaged DNA-based messages into recipient fibroblasts and are involved in various cell-related processes in the recipient fibroblasts by regulating gene expression (Waldenström et al., 2012). MiR-30a, which is derived from hypoxic cardiomyocytes, is efficiently transferred between cardiomyocytes via exosomes, and regulates autophagy by affecting the expression of Beclin1, ATG12, and the ratio of LC3II/LC3I, which are important regulators of autophagy (Yang et al., 2016). Yang et al. (2018) also confirmed that cardiomyocyte-derived exosomes can be absorbed by fibroblasts, and that these exosomes transfer miR208a between myocytes and fibroblasts. Their data suggested that crosstalk between myocytes and fibroblasts via exosomes contributed to the development of myocardial fibrosis. Recently, it was reported that injured cardiomyocyte-derived exosomes accelerated the injury of bone marrow-derived mesenchymal stem cells transplanted into a heart infarction mouse model 


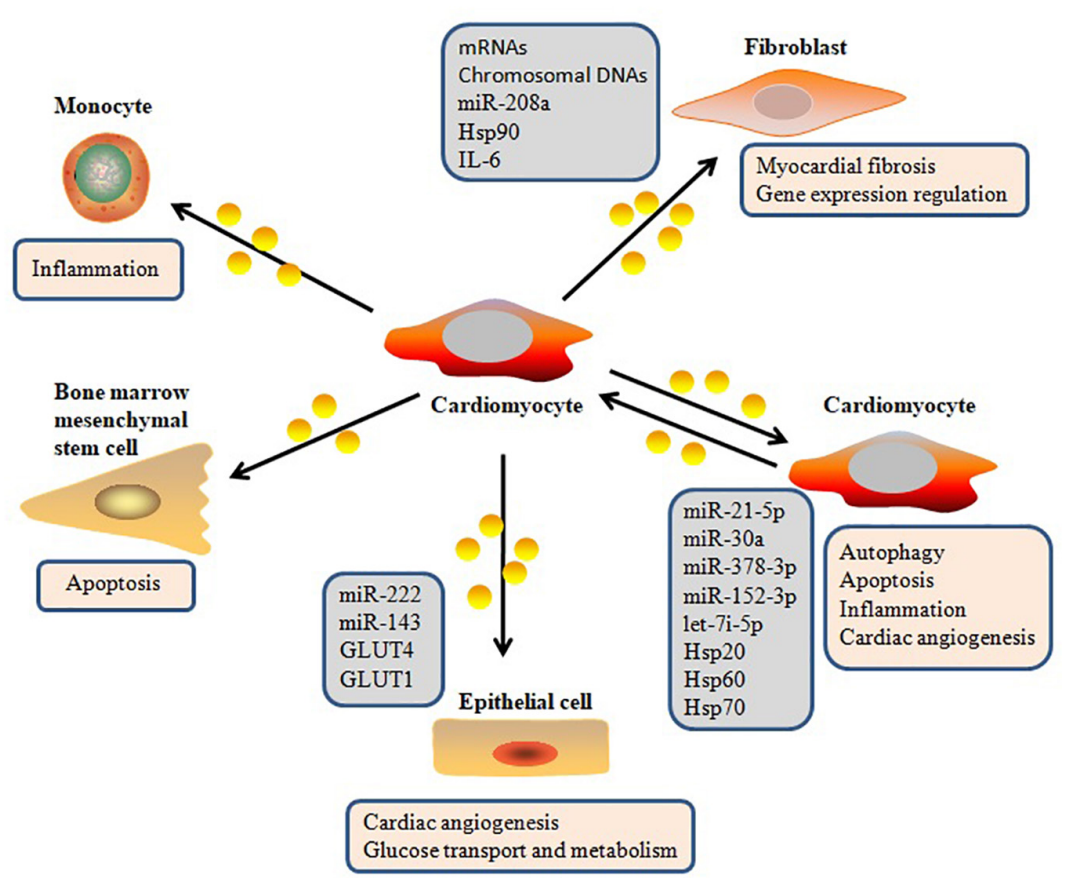

FIGURE 2 | Exosome-mediated cell-cell communication in heart.

by mediating cell-cell communication (Hu et al., 2018). Recent research has described the molecular mechanisms of the intracellular autophagy pathway induced by exosomes, which delivers autophagy-associated molecules (Zheng et al., 2019). Bollini et al. (2018) reported that exosomes play significant roles in intercellular signaling, and that cardiomyocyte-derived exosomes affect the function of other cardiac cell types, thereby influencing many physiological and pathological functions of the heart. Yuan et al. (2016) also reported that exosomes from cardiomyocytes mediated cardiac repair after myocardial infarction by delivering a variety of functional molecules into their target cells. Ribeiro et al. (2013) further found that exosomes secreted by cardiomyocytes can carry and transfer proangiogenic and anti-angiogenic factors, indicating that they play a role in regulating angiogenesis. Taken together, recent evidence has established that exosomes secreted from cardiomyocytes can deliver a wide variety of biomolecules into other cell types and regulate gene expression in these cells.

It should be noted that the presence and relative abundance of biomolecules inside or on the surface of exosomes can fluctuate depending on the cell type and pathophysiological state (Zhou et al., 2006; Cosme et al., 2017; da Silva Novaes et al., 2019). Recently, Kuo et al. (2019) reported that simvastatin, a potent competitive inhibitor of 5-hydroxy-3-methylglutaryl-coenzyme A reductase, attenuated collagen-associated protein expression in cardiomyocyte-derived exosomes and reduced the uptake of the exosomes by human cardiac fibroblasts. Yang et al. (2018) also found that, in a rat model of cardiac fibrosis, miR-208a levels were up-regulated in cardiomyocyte-derived exosomes. The onset of myocardial infarction also induced the release of miRNA-enriched exosomes (Abreu and da Costa Martins, 2018).
Additionally, diabetic cardiomyocytes exhibited increased secretion of detrimental exosomes containing decreased Hsp20 levels, which contributed to diabetes-induced organ damage (Wang et al., 2016).

\section{POTENTIAL DIAGNOSTIC AND THERAPEUTIC APPLICATIONS OF CARDIOMYOCYTE-DERIVED EXOSOMES}

Emerging evidence shows that exosomes have great diagnostic and therapeutic potential (Cobelli et al., 2017; Kim and Kim, 2017; Campanella et al., 2019; Urban et al., 2019). The remarkable diagnostic and therapeutic potential of exosomes in CVDs has been increasingly investigated in recent years (Bei et al., 2017b; Sun et al., 2017; Ghafarian et al., 2018). A large number of studies have confirmed the diagnostic and therapeutic applications of exosomes derived from cardiac fibroblasts, cardiac telocytes, cardiosphere-derived cells, and cardiac progenitor and stem cells in terms of CVD (Bang et al., 2014; Barile et al., 2014; Fertig et al., 2014; Khan et al., 2015; Lyu et al., 2015; Kervadec et al., 2016; Xiao et al., 2016; Agarwal et al., 2017; Gallet et al., 2017; Barile et al., 2018; Gao et al., 2018; Ju et al., 2018; Namazi et al., 2018; Zhu et al., 2018). Recent findings have confirmed the important role of cardiomyocyte-derived exosomes in the diagnosis, prognosis, and therapy of various diseases. Exosomes secreted from dystrophin-deficiency-induced pluripotent stem cell-derived cardiomyocytes exert cardioprotection via the presence of exosomal surface proteins and the activation of 
ERK1/2 and p38MAPK signaling (Gartz et al., 2018). Upon myocardial infarction, cardiomyocyte-derived exosomes were shown to regulate local inflammatory responses through the stimulation of cardiac monocytes (Loyer et al., 2018). Therefore, it is thought that the exosome is an important component of the cardiac microenvironment and plays a complex role in the treatment of myocardial infarction. Similarly, exosomes secreted from injured cardiomyocytes were found to exert negative effects similar to those of cardiomyocyte-derived exosomes in myocardial infarction ( $\mathrm{Hu}$ et al., 2018). Cardiomyocytederived exosomes also mediate intercellular communication for myocardial repair and regeneration (Ong et al., 2018). Studies have demonstrated that dysregulated miRNAs contained in cardiomyocyte-derived exosomes, such as let-7i-5p, miR21-5p, miR-27a, miR-28-3p, miR-34a, miR-143, miR-222, and miR-378-3p, regulate cardiac function by targeting different mRNAs (Ribeiro-Rodrigues et al., 2017; Zhang et al., 2017; Tian et al., 2018). These findings will accelerate the development of promising therapeutic strategies for patients with ischemic heart disease and chronic heart failure. Further studies are urgently needed to explore the possible molecular mechanisms underlying the selective loading and accumulation of certain molecules, substrate specificity, and the regulation of target cell by exosomes (Jansen and Li, 2017; Vanhaverbeke et al., 2017).

\section{CONCLUSION AND FUTURE PERSPECTIVES}

The field of exosome analysis has expanded greatly in recent years. In this review, we concentrated on the biological functions

\section{REFERENCES}

Abreu, R. C., and da Costa Martins, P. A. (2018). cROSsing the cardiac MIRe: fibroblast-cardiomyocyte ex(o)press. Am. J. Physiol. Heart Circ. Physiol. 314, H1253-H1255. doi: 10.1152/ajpheart.00096.2018

Agarwal, U., George, A., Bhutani, S., Ghosh-Choudhary, S., Maxwell, J. T., Brown, M. E., et al. (2017). Experimental, systems, and computational approaches to understanding the microRNA-mediated reparative potential of cardiac progenitor cell-derived exosomes from pediatric patients. Circ. Res. 120, 701-712. doi: 10.1161/CIRCRESAHA.116.309935

Akers, J. C., Gonda, D., Kim, R., Carter, B. S., and Chen, C. C. (2013). Biogenesis of extracellular vesicles (EV): exosomes, microvesicles, retrovirus-like vesicles, and apoptotic bodies. J. Neurooncol. 113, 1-11. doi: 10.1007/s11060-013-1084-8

Bang, C., Batkai, S., Dangwal, S., Gupta, S. K., Foinquinos, A., Holzmann, A., et al. (2014). Cardiac fibroblast-derived microRNA passenger strand-enriched exosomes mediate cardiomyocyte hypertrophy. J. Clin. Invest. 124, 2136-2146. doi: 10.1172/JCI70577

Barile, L., Cervio, E., Lionetti, V., Milano, G., Ciullo, A., Biemmi, V., et al. (2018). Cardioprotection by cardiac progenitor cell-secreted exosomes: role of pregnancy-associated plasma protein-A. Cardiovasc. Res. 114, 992-1005. doi: $10.1093 / \mathrm{cvr} / \mathrm{cvy} 055$

Barile, L., Lionetti, V., Cervio, E., Matteucci, M., Gherghiceanu, M., Popescu, L. M., et al. (2014). Extracellular vesicles from human cardiac progenitor cells inhibit cardiomyocyte apoptosis and improve cardiac function after myocardial infarction. Cardiovasc. Res. 103, 530-541. doi: 10.1093/cvr/cvu167

Barlow, J. P., and Solomon, T. P. (2018). Do skeletal muscle-secreted factors influence the function of pancreatic $\beta$-cells? Am. J. Physiol. Endocrinol. Metab. 314, E297-E307. doi: 10.1152/ajpendo.00353.2017 and potential therapeutic implications of cardiomyocyte-derived exosomes, including biogenesis, molecular contents, isolation techniques, biomarkers, cardiovascular cell-cell communication, and potential diagnostic and therapeutic applications of various diseases.

Although exosome biology represents a fascinating area for future therapeutic cardioprotection strategies, there remain several technical challenges to this field of research. The current major technical challenge is the ability to differentiate exosomes derived from cells under normal and pathological conditions for therapeutic applications. Another challenge is the application of exosomal research methodologies and tools in vivo. Once these challenges are overcome, new techniques for the efficient isolation, quantification, and analysis of exosomes will be helpful to elucidate the function and influence of exosomes in the cardiac microenvironment in vivo, and will facilitate the development of new therapeutic strategies to efficiently diagnose and treat heart diseases.

\section{AUTHOR CONTRIBUTIONS}

Both authors listed have made a substantial, direct and intellectual contribution to the work, and approved it for publication.

\section{FUNDING}

HY was supported by research grants from the National Natural Science Foundation of China (81560057 and 81760056).

Bei, Y., Chen, T., Banciu, D. D., Cretoiu, D., and Xiao, J. (2017a). Circulating exosomes in cardiovascular diseases. Adv. Exp. Med. Biol. 998, 255-269. doi: 10.1007/978-981-10-4397-0_17

Bei, Y., Das, S., Rodosthenous, R. S., Holvoet, P., Vanhaverbeke, M., Monteiro, M. C., et al. (2017b). Extracellular vesicles in cardiovascular theranostics. Theranostics 7, 4168-4182. doi: 10.7150/thno.21274

Bollini, S., Smits, A. M., Balbi, C., Lazzarini, E., and Ameri, P. (2018). Triggering endogenous cardiac repair and regeneration via extracellular vesicle-mediated communication. Front. Physiol. 9:1497. doi: 10.3389/fphys.2018.01497

Brisson, A. R., Tan, S., Linares, R., Gounou, C., and Arraud, N. (2017). Extracellular vesicles from activated platelets: a semiquantitative cryo-electron microscopy and immuno-gold labeling study. Platelets 28, 263-271. doi: 10.1080/09537104. 2016

Campanella, C., Caruso Bavisotto, C., Logozzi, M., Marino Gammazza, A., Mizzoni, D., Cappello, F., et al. (2019). On the choice of the extracellular vesicles for therapeutic purposes. Int. J. Mol. Sci. 20:236. doi: 10.3390/ijms20020236

Cervio, E., Barile, L., Moccetti, T., and Vassalli, G. (2015). Exosomes for intramyocardial intercellular communication. Stem Cells Int. 2015:482171. doi: $10.1155 / 2015 / 482171$

Chaturvedi, P., Kalani, A., Medina, I., Familtseva, A., and Tyagi, S. C. (2015). Cardiosome mediated regulation of MMP9 in diabetic heart: role of mir29b and mir455 in exercise. J. Cell Mol. Med. 19, 2153-2161. doi: 10.1111/jcmm.12589

Cheng, L., Zhao, W., and Hill, A. F. (2018). Exosomes and their role in the intercellular trafficking of normal and disease associated prion proteins. Mol. Aspects Med. 60, 62-68. doi: 10.1016/j.mam.2017.11.011

Chistiakov, D. A., Orekhov, A. N., and Bobryshev, Y. V. (2016). Cardiac extracellular vesicles in normal and infarcted heart. Int. J. Mol. Sci. 17:E63. doi: 10.3390/ijms17010063 
Cobelli, N. J., Leong, D. J., and Sun, H. B. (2017). Exosomes: biology, therapeutic potential, and emerging role in musculoskeletal repair and regeneration. Ann. N. Y. Acad. Sci. 1410, 57-67. doi: 10.1111/nyas.13469

Conigliaro, A., Fontana, S., Raimondo, S., and Alessandro, R. (2017). Exosomes: nanocarriers of biological messages. Adv. Exp. Med. Biol. 998, 23-43. doi: 10. 1007/978-981-10-4397-0_2

Cosme, J., Guo, H., Hadipour-Lakmehsari, S., Emili, A., and Gramolini, A. O. (2017). Hypoxia-induced changes in the fibroblast secretome, exosome, and whole-cell proteome using cultured, cardiac-derived cells isolated from neonatal mice. J. Proteome Res. 16, 2836-2847. doi: 10.1021/acs.jproteome. $7 \mathrm{~b} 00144$

da Silva Novaes, A., Borges, F. T., Maquigussa, E., Varela, V. A., Dias, M. V. S., and Boim, M. A. (2019). Influence of high glucose on mesangial cell-derived exosome composition, secretion and cell communication. Sci. Rep. 9:6270. doi: 10.1038/s41598-019-42746-1

Datta, R., Bansal, T., Rana, S., Datta, K., Datta Chaudhuri, R., Chawla-Sarkar, M., et al. (2017). Myocyte-derived Hsp90 modulates collagen upregulation via biphasic activation of STAT-3 in fibroblasts during cardiac hypertrophy. Mol. Cell Biol. 37, e00611-e00616. doi: 10.1128/MCB.00611-16

Davidson, S. M., and Yellon, D. M. (2018). Exosomes and cardioprotection A critical analysis. Mol. Aspects Med. 60, 104-114. doi: 10.1016/j.mam.2017. 11.004

Enderle, D., Spiel, A., Coticchia, C. M., Berghoff, E., Mueller, R., Schlumpberger, M., et al. (2015). Characterization of RNA from exosomes and other extracellular vesicles isolated by a novel spin column-based method. PLoS One 10:e0136133. doi: 10.1371/journal.pone.0136133

Feng, Y., Huang, W., Meng, W., Jegga, A. G., Wang, Y., Cai, W., et al. (2014). Heat shock improves Sca-1+ stem cell survival and directs ischemic cardiomyocytes toward a prosurvival phenotype via exosomal transfer: a critical role for HSF1/miR-34a/HSP70 pathway. Stem Cells 32, 462-472. doi: 10.1002/stem. 1571

Fertig, E. T., Gherghiceanu, M., and Popescu, L. M. (2014). Extracellular vesicles release by cardiac telocytes: electron microscopy and electron tomography. J. Cell Mol. Med. 18, 1938-1943. doi: 10.1111/jcmm.12436

Fruhbeis, C., Frohlich, D., and Kramer-Albers, E. M. (2012). Emerging roles of exosomes in neuron-glia communication. Front. Physiol. 3:119. doi: 10.3389/ fphys.2012.00119

Frydrychowicz, M., Kolecka-Bednarczyk, A., Madejczyk, M., Yasar, S., and Dworacki, G. (2015). Exosomes - structure, biogenesis and biological role in non-small-cell lung cancer. Scand. J. Immunol. 81, 2-10. doi: 10.1111/sji.12247

Gallet, R., Dawkins, J., Valle, J., Simsolo, E., de Couto, G., Middleton, R., et al. (2017). Exosomes secreted by cardiosphere-derived cells reduce scarring, attenuate adverse remodelling, and improve function in acute and chronic porcine myocardial infarction. Eur. Heart J. 38, 201-211. doi: 10.1093/ eurheartj/ehw2 40

Gao, L., Gregorich, Z. R., Zhu, W., Mattapally, S., Oduk, Y., Lou, X., et al. (2018). Large cardiac muscle patches engineered from human induced-pluripotent stem cell-derived cardiac cells improve recovery from myocardial infarction in Swine. Circulation 137, 1712-1730. doi: 10.1161/CIRCULATIONAHA.117. 030785

Garcia, N. A., Moncayo-Arlandi, J., Sepulveda, P., and Diez-Juan, A. (2016). Cardiomyocyte exosomes regulate glycolytic flux in endothelium by direct transfer of GLUT transporters and glycolytic enzymes. Cardiovasc. Res. 109, 397-408. doi: 10.1093/cvr/cvv260

Garcia, N. A., Ontoria-Oviedo, I., González-King, H., Diez-Juan, A., and Sepúlveda, P. (2015). Glucose starvation in cardiomyocytes enhances exosome secretion and promotes angiogenesis in endothelial cells. PLoS One 10:e0138849. doi: 10.1371/journal.pone.0138849

Gartz, M., Darlington, A., Afzal, M. Z., and Strande, J. L. (2018). Exosomes exert cardioprotection in dystrophin-deficient cardiomyocytes via ERK1/2p38/MAPK signaling. Sci. Rep. 8:16519. doi: 10.1038/s41598-018-34879-6

GBD 2013 Mortality and Causes of Death Collaborators, (2015). Global, regional, and national age-sex specific all-cause and cause-specific mortality for 240 causes of death, 1990-2013: a systematic analysis for the Global Burden of Disease Study 2013. Lancet. 385, 117-171. doi: 10.1016/S0140-6736(14)61 682-2

Gennebäck, N., Hellman, U., Malm, L., Larsson, G., Ronquist, G., Waldenström, A., et al. (2013). Growth factor stimulation of cardiomyocytes induces changes in the transcriptional contents of secreted exosomes. J. Extracell Vesicles 2, doi: 10.3402/jev.v2i0.20167

Ghafarian, F., Pashirzad, M., Khazaei, M., Rezayi, M., Hassanian, S. M., Ferns, G. A., et al. (2018). The clinical impact of exosomes in cardiovascular disorders: from basic science to clinical application. J. Cell Physiol. 234, 12226-12236. doi: $10.1002 /$ jcp. 27964

Gupta, S., and Knowlton, A. A. (2007). HSP60 trafficking in adult cardiac myocytes: role of the exosomal pathway. Am. J. Physiol. Heart Circ. Physiol. 292, H3052-H3056. doi: 10.1152/ajpheart.01355.2006

Gurunathan, S., Kang, M. H., Jeyaraj, M., Qasim, M., and Kim, J. H. (2019). Review of the isolation, characterization, biological function, and multifarious therapeutic approaches of exosomes. Cells 8:E307. doi: 10.3390/cells8040307

György, B., Szabó, T. G., Pásztói, M., Pál, Z., Misják, P., Aradi, B., et al. (2011). Membrane vesicles, current state-of-the-art: emerging role of extracellular vesicles. Cell Mol. Life Sci. 68, 2667-2688. doi: 10.1007/s00018-011-0689-3

Hu, M., Guo, G., Huang, Q., Cheng, C., Xu, R., Li, A., et al. (2018). The harsh microenvironment in infarcted heart accelerates transplanted bone marrow mesenchymal stem cells injury: the role of injured cardiomyocytes-derived exosomes. Cell Death Dis. 9:357. doi: 10.1038/s41419-018-0392-5

Ibrahim, A. G., Cheng, K., and Marbán, E. (2014). Exosomes as critical agents of cardiac regeneration triggered by cell therapy. Stem Cell Rep. 2, 606-619. doi: 10.1016/j.stemcr.2014.04.006

Jakhar, R., and Crasta, K. (2019). Exosomes as emerging pro-tumorigenic mediators of the senescence-associated secretory phenotype. Int. J. Mol. Sci. 20:E2547. doi: 10.3390/ijms20102547

Jansen, F., and Li, Q. (2017). Exosomes as diagnostic biomarkers in cardiovascular diseases. Adv. Exp. Med. Biol. 998, 61-70. doi: 10.1007/978-981-10-4397-0_4

Johnstone, R. M., Adam, M., Hammond, J. R., Orr, L., and Turbide, C. (1987). Vesicle formation during reticulocyte maturation. Association of plasma membrane activities with released vesicles (exosomes). J. Biol. Chem. 262, 9412-9420.

Ju, C., Shen, Y., Ma, G., Liu, Y., Cai, J., Kim, I. M., et al. (2018). Transplantation of cardiac mesenchymal stem cell-derived exosomes promotes repair in ischemic myocardium. J. Cardiovasc. Transl. Res. 11, 420-428. doi: 10.1007/s12265-0189822-0

Juni, R. P., Abreu, R. C., and da Costa Martins, P. A. (2017). Regulation of microvascularization in heart failure - an endothelial cell, non-coding RNAs and exosome liaison. Noncoding RNA Res. 2, 45-55. doi: 10.1016/j.ncrna.2017. 01.001

Katsuda, T., Kosaka, N., and Ochiya, T. (2014). The roles of extracellular vesicles in cancer biology: toward the development of novel cancer biomarkers. Proteomics 14, 412-425. doi: 10.1002/pmic.201300389

Kervadec, A., Bellamy, V., El Harane, N., Arakélian, L., Vanneaux, V., Cacciapuoti, I., et al. (2016). Cardiovascular progenitor-derived extracellular vesicles recapitulate the beneficial effects of their parent cells in the treatment of chronic heart failure. J. Heart Lung Transplant. 35, 795-807. doi: 10.1016/j.healun.2016. 01.013

Khan, M., Nickoloff, E., Abramova, T., Johnson, J., Verma, S. K., Krishnamurthy, P., et al. (2015). Embryonic stem cell-derived exosomes promote endogenous repair mechanisms and enhance cardiac function following myocardial infarction. Circ. Res. 117, 52-64. doi: 10.1161/CIRCRESAHA.117.30 5990

Kim, S. M., and Kim, H. S. (2017). Engineering of extracellular vesicles as drug delivery vehicles. Stem Cell Investig. 4:74. doi: 10.21037/sci.2017.08.07

Kishore, R., Garikipati, V. N., and Gumpert, A. (2016). Tiny shuttles for information transfer: exosomes in cardiac health and disease. J. Cardiovasc. Transl. Res. 9, 169-175. doi: 10.1007/s12265-016-9682-4

Kowal, J., Tkach, M., and Théry, C. (2014). Biogenesis and secretion of exosomes. Curr. Opin. Cell Biol. 29, 116-125. doi: 10.1016/j.ceb.2014.05.004

Kuo, H. F., Hsieh, C. C., Wang, S. C., Chang, C. Y., Hung, C. H., Kuo, P. L., et al. (2019). Simvastatin attenuates cardiac fibrosis via regulation of cardiomyocytederived exosome secretion. J. Clin. Med. 8:E794. doi: 10.3390/jcm8060794

Lai, S. L., Marín-Juez, R., and Stainier, D. Y. R. (2019). Immune responses in cardiac repair and regeneration: a comparative point of view. Cell Mol. Life Sci. 76, 1365-1380. doi: 10.1007/s00018-018-2995-5

Lobb, R. J., Becker, M., Wen, S. W., Wong, C. S., Wiegmans, A. P., Leimgruber, A., et al. (2015). Optimized exosome isolation protocol for cell culture supernatant and human plasma. J. Extracell. Vesicles. 4:27031. doi: 10.3402/jev.v4.27031 
Loyer, X., Zlatanova, I., Devue, C., Yin, M., Howangyin, K. Y., Klaihmon, P., et al. (2018). Intra-cardiac release of extracellular vesicles shapes inflammation following myocardial infarction. Circ. Res. 123, 100-106. doi: 10.1161/ CIRCRESAHA.117.311326

Lyu, L., Wang, H., Li, B., Qin, Q., Qi, L., Nagarkatti, M., et al. (2015). A critical role of cardiac fibroblast-derived exosomes in activating renin angiotensin system in cardiomyocytes. J. Mol. Cell Cardiol. 89, 268-279. doi: 10.1016/j.yjmcc.2015. 10.022

Macías, M., Rebmann, V., Mateos, B., Varo, N., Perez-Gracia, J. L., Alegre, E., et al. (2019). Comparison of six commercial serum exosome isolation methods suitable for clinical laboratories. Effect in cytokine analysis. Clin. Chem. Lab. Med. doi: 10.1515/cclm-2018-1297 [Epub ahead of print].

Malik, Z. A., Kott, K. S., Poe, A. J., Kuo, T., Chen, L., Ferrara, K. W., et al. (2013). Cardiac myocyte exosomes: stability, HSP60, and proteomics. Am. J. Physiol. Heart Circ. Physiol. 304, H954-H965. doi: 10.1152/ajpheart.00835.2012

Malik, Z. A., Liu, T. T., and Knowlton, A. A. (2016). Cardiac myocyte exosome isolation. Methods Mol. Biol. 1448, 237-248. doi: 10.1007/978-1-4939-375 3-0_17

Marini, M., Ibba-Manneschi, L., and Manetti, M. (2017). Cardiac telocyte-derived exosomes and their possible implications in cardiovascular pathophysiology. Adv. Exp. Med. Biol. 998, 237-254. doi: 10.1007/978-981-10-4397-0_16

Mathivanan, S., and Simpson, R. J. (2009). ExoCarta: a compendium of exosomal proteins and RNA. Proteomics 9, 4997-5000. doi: 10.1002/pmic.200900351

Meng, W., Hao, Y., He, C., Li, L., and Zhu, G. (2019). Exosome-orchestrated hypoxic tumor microenvironment. Mol. Cancer 18:57. doi: 10.1186/s12943019-0982-6

Momen-Heravi, F., Balaj, L., Alian, S., Trachtenberg, A. J., Hochberg, F. H., Skog, J., et al. (2012). Impact of biofluid viscosity on size and sedimentation efficiency of the isolated microvesicles. Front. Physiol. 3:162. doi: 10.3389/fphys.2012. 00162

Namazi, H., Mohit, E., Namazi, I., Rajabi, S., Samadian, A., Hajizadeh-Saffar, E., et al. (2018). Exosomes secreted by hypoxic cardiosphere-derived cells enhance tube formation and increase pro-angiogenic miRNA. J. Cell Biochem. 119, 4150-4160. doi: 10.1002/jcb.26621

Ohno, S., and Kuroda, M. (2016). Exosome-mediated targeted delivery of miRNAs. Methods Mol. Biol. 1448, 261-270. doi: 10.1007/978-1-4939-3753-0_19

Ong, S. G., Lee, W. H., Zhou, Y., and Wu, J. C. (2018). Mining exosomal microRNAs from human-induced pluripotent stem cells-derived cardiomyocytes for cardiac regeneration. Methods Mol. Biol. 1733, 127-136. doi: 10.1007/978-1-4939-7601-0_10

Pegtel, D. M., Cosmopoulos, K., Thorley-Lawson, D. A., van Eijndhoven, M. A., Hopmans, E. S., Lindenberg, J. L., et al. (2010). Functional delivery of viral miRNAs via exosomes. Proc. Natl. Acad. Sci. U.S.A. 107, 6328-6333. doi: 10. 1073/pnas.0914843107

Peterson, M. F., Otoc, N., Sethi, J. K., Gupta, A., and Antes, T. J. (2015). Integrated systems for exosome investigation. Methods 87, 31-45. doi: 10.1016/j.ymeth. 2015.04.015

Poe, A. J., and Knowlton, A. A. (2017). Exosomes as agents of change in the cardiovascular system. J. Mol. Cell Cardiol. 111, 40-50. doi: 10.1016/j.yjmcc. 2017.08.002

Poe, A. J., and Knowlton, A. A. (2018). Exosomes and cardiovascular cell-cell communication. Essays Biochem. 62, 193-204. doi: 10.1042/EBC20170081

Prunotto, M., Farina, A., Lane, L., Pernin, A., Schifferli, J., Hochstrasser, D. F., et al. (2013). Proteomic analysis of podocyte exosome-enriched fraction from normal human urine. J. Proteomics 82, 193-229. doi: 10.1016/j.jprot.2013.01.012

Ribeiro, M. F., Zhu, H., Millard, R. W., and Fan, G. C. (2013). Exosomes function in pro- and anti-angiogenesis. Curr. Angiogenes. 2, 54-59. doi: 10. 2174/22115528113020020001

Ribeiro-Rodrigues, T. M., Laundos, T. L., Pereira-Carvalho, R., BatistaAlmeida, D., Pereira, R., Coelho-Santos, V., et al. (2017). Exosomes secreted by cardiomyocytes subjected to ischaemia promote cardiac angiogenesis. Cardiovasc. Res. 113, 1338-1350. doi: 10.1093/cvr/cvx118

Salem, E. S. B., and Fan, G. C. (2017). Pathological effects of exosomes in mediating diabetic cardiomyopathy. Adv. Exp. Med. Biol. 998, 113-138. doi: 10.1007/978981-10-4397-0_8

Scherer, P. E. (2019). The many secret lives of adipocytes: implications for diabetes. Diabetologia 62, 223-232. doi: 10.1007/s00125-018-4777-x
Seo, Y., Kim, H. S., and Hong, I. S. (2019). Stem cell-derived extracellular vesicles as immunomodulatory therapeutics. Stem Cells Int. 2019, 5126156. doi: 10.1155/ 2019/5126156

Sluijter, J. P., Verhage, V., Deddens, J. C., van den Akker, F., and Doevendans, P. A. (2014). Microvesicles and exosomes for intracardiac communication. Cardiovasc. Res. 102, 302-311. doi: 10.1093/cvr/cvu022

Stoorvogel, W., Kleijmeer, M. J., Geuze, H. J., and Raposo, G. (2002). The biogenesis and functions of exosomes. Traffic 3, 321-330. doi: 10.1034/j.16000854.2002.30502.x

Sun, H. J., Zhu, X. X., Cai, W. W., and Qiu, L. Y. (2017). Functional roles of exosomes in cardiovascular disorders: a systematic review. Eur. Rev. Med. Pharmacol.Sci. 21, 5197-5206. doi: 10.26355/eurrev_201711_13840

Talman, V., and Kivelä, R. (2018). Cardiomyocyte-endothelial cell interactions in cardiac remodeling and regeneration. Front. Cardiovasc. Med. 5:101. doi: 10.3389/fcvm.2018.00101

Tian, C., Gao, L., Zimmerman, M. C., and Zucker, I. H. (2018). Myocardial infarction-induced microRNA-enriched exosomes contribute to cardiac Nrf2 dysregulation in chronic heart failure. Am. J. Physiol. Heart Circ. Physiol. 314, H928-H939. doi: 10.1152/ajpheart.00602.2017

Torralba, D., Martín-Cófreces, N. B., and Sanchez-Madrid, F. (2019). Mechanisms of polarized cell-cell communication of T lymphocytes. Immunol. Lett. 209, 11-20. doi: 10.1016/j.imlet.2019.03.009

Trajkovic, K., Hsu, C., Chiantia, S., Rajendran, L., Wenzel, D., Wieland, F., et al. (2008). Ceramide triggers budding of exosome vesicles into multivesicular endosomes. Science 319, 1244-1247. doi: 10.1126/science.1153124

Urban, S. K., Mocan, T., Sänger, H., Lukacs-Kornek, V., and Kornek, M. (2019). Extracellular vesicles in liver diseases: diagnostic, prognostic, and therapeutic application. Semin. Liver Dis. 39, 70-77. doi: 10.1055/s-0038-1676122

Van Deun, J., Mestdagh, P., Sormunen, R., Cocquyt, V., Vermaelen, K., Vandesompele, J., et al. (2014). The impact of disparate isolation methods for extracellular vesicles on downstream RNA profiling. J. Extracell. Vesicles 3, doi: $10.3402 /$ jev.v3.24858

Vanhaverbeke, M., Gal, D., and Holvoet, P. (2017). Functional role of cardiovascular exosomes in myocardial injury and atherosclerosis. Adv. Exp. Med. Biol. 998, 45-58. doi: 10.1007/978-981-10-4397-0_3

Waldenström, A., Gennebäck, N., Hellman, U., and Ronquist, G. (2012). Cardiomyocyte microvesicles contain DNA/RNA and convey biological messages to target cells. PLoS One 7:e34653. doi: 10.1371/journal.pone.0034653

Wang, X., Gu, H., Huang, W., Peng, J., Li, Y., Yang, L., et al. (2016). Hsp20mediated activation of exosome biogenesis in cardiomyocytes improves cardiac function and angiogenesis in diabetic mice. Diabetes 65, 3111-3128. doi: 10 . 2337/db15-1563

Wang, X., Huang, W., Liu, G., Cai, W., Millard, R. W., Wang, Y., et al. (2014). Cardiomyocytes mediate anti-angiogenesis in type 2 diabetic rats through the exosomal transfer of miR-320 into endothelial cells. J. Mol. Cell Cardiol. 74, 139-150. doi: 10.1016/j.yjmcc.2014.05.001

Wang, Z., Wu, H. J., Fine, D., Schmulen, J., Hu, Y., Godin, B., et al. (2013). Ciliated micropillars for the microfluidic-based isolation of nanoscale lipid vesicles. $L a b$. Chip. 13, 2879-2882. doi: 10.1039/c3lc41343h

Witwer, K. W., Buzás, E. I., Bemis, L. T., Bora, A., Lässer, C., Lötvall, J., et al. (2013). Standardization of sample collection, isolation and analysis methods in extracellular vesicle research. J. Extracell. Vesicles 2. doi: 10.3402/jev.v2i0.20360

$\mathrm{Wu}, \mathrm{R} ., \mathrm{Gao}, \mathrm{W}$. Yao, K., and Ge, J. (2019). Roles of exosomes derived from immune cells in cardiovascular diseases. Front. Immunol. 10:648. doi: 10.3389/ fimmu.2019.00648

Xiao, J., Pan, Y., Li, X. H., Yang, X. Y., Feng, Y. L., Tan, H. H., et al. (2016). Cardiac progenitor cell-derived exosomes prevent cardiomyocytes apoptosis through exosomal miR-21 by targeting PDCD4. Cell Death Dis. 7:e2277. doi: 10.1038/cddis.2016.181

Yang, J., Yu, X., Xue, F., Li, Y., Liu, W., and Zhang, S. (2018). Exosomes derived from cardiomyocytes promote cardiac fibrosis via myocyte-fibroblast crosstalk. Am. J. Transl. Res. 10, 4350-4366.

Yang, Y., Li, Y., Chen, X., Cheng, X., Liao, Y., and Yu, X. (2016). Exosomal transfer of miR-30a between cardiomyocytes regulates autophagy after hypoxia. J. Mol. Med. 94, 711-724. doi: 10.1007/s00109-016-1387-2

You, B., Xu, W., and Zhang, B. (2018). Engineering exosomes: a new direction for anticancer treatment. Am. J. Cancer Res. 8, 1332-1342. 
Yu, D. W., Ge, P. P., Liu, A. L., Yu, X. Y., and Liu, T. T. (2019). HSP20-mediated cardiomyocyte exosomes improve cardiac function in mice with myocardial infarction by activating Akt signaling pathway. Eur. Rev. Med. Pharmacol. Sci. 23, 4873-4881. doi: 10.26355/eurrev_201906_18075

Yu, X., Deng, L., Wang, D., Li, N., Chen, X., Cheng, X., et al. (2012). Mechanism of TNF- $\alpha$ autocrine effects in hypoxic cardiomyocytes: initiated by hypoxia inducible factor $1 \alpha$, presented by exosomes. J. Mol. Cell Cardiol. 53, 848-857. doi: 10.1016/j.yjmcc.2012.10.002

Yuan, M. J., Maghsoudi, T., and Wang, T. (2016). Exosomes mediate the intercellular communication after myocardial infarction. Int. J. Med. Sci. 13, 113-116. doi: 10.7150/ijms.14112

Zarovni, N., Corrado, A., Guazzi, P., Zocco, D., Lari, E., Radano, G., et al. (2015). Integrated isolation and quantitative analysis of exosome shuttled proteins and nucleic acids using immunocapture approaches. Methods 87, 46-58. doi: 10.1016/j.ymeth.2015.05.028

Zeringer, E., Barta, T., Li, M., and Vlassov, A. V. (2015). Strategies for isolation of exosomes. Cold Spring Harb. Protoc. 2015, 319-323. doi: 10.1101/pdb. top074476

Zhang, J., Ma, J., Long, K., Qiu, W., Wang, Y., Hu, Z., et al. (2017). Overexpression of exosomal cardioprotective miRNAs mitigates hypoxia-induced H9c2 cells apoptosis. Int. J. Mol. Sci. 18:E711. doi: 10.3390/ijms18040711

Zhang, X., Wang, X., Zhu, H., Kranias, E. G., Tang, Y., Peng, T., et al. (2012). Hsp20 functions as a novel cardiokine in promoting angiogenesis via activation of VEGFR2. PLoS One 7:e32765. doi: 10.1371/journal.pone.0032765
Zheng, J., Tan, J., Miao, Y. Y., and Zhang, Q. (2019). Extracellular vesicles degradation pathway based autophagy lysosome pathway. Am. J. Transl. Res. 11, 1170-1183.

Zhou, H., Pisitkun, T., Aponte, A., Yuen, P. S., Hoffert, J. D., Yasuda, H., et al. (2006). Exosomal fetuin-A identified by proteomics: a novel urinary biomarker for detecting acute kidney injury. Kidney Int. 70, 1847-1857. doi: 10.1038/sj.ki. 5001874

Zhu, J., Lu, K., Zhang, N., Zhao, Y., Ma, Q., Shen, J., et al. (2018). Myocardial reparative functions of exosomes from mesenchymal stem cells are enhanced by hypoxia treatment of the cells via transferring microRNA-210 in an nSMase2-dependent way. Artif. Cells Nanomed. Biotechnol. 46, 1659-1670. doi: 10.1080/21691401.2017.138 8249

Conflict of Interest Statement: The authors declare that the research was conducted in the absence of any commercial or financial relationships that could be construed as a potential conflict of interest.

Copyright (c) 2019 Yu and Wang. This is an open-access article distributed under the terms of the Creative Commons Attribution License (CC BY). The use, distribution or reproduction in other forums is permitted, provided the original author(s) and the copyright owner(s) are credited and that the original publication in this journal is cited, in accordance with accepted academic practice. No use, distribution or reproduction is permitted which does not comply with these terms. 